\title{
NOVEL PROMISING INHIBITORS OF TYROSYL- DNA PHOSPHODIESTERASE I (TDP 1) COMBINING 4-ARYLCOUMARIN AND MONOTERPENOID MOIETIES
}

\section{T. Khomenko', A. Zakharenko², A. Chepanova², E. Ilina², O. Zakharova², V. Kaledin', V. Nikolin', N. Popova³, D. Korchagina', K. Volcho', N. Salakhutdinov' and O. Lavrik ${ }^{2}$}

${ }^{1}$ N.N. Vorozhtsov Novosibirsk Institute of Organic Chemistry, Siberian Branch of the Russian Academy of Sciences, 630090, Russia, Novosibirsk, pr. Lavrentieva, 9. ${ }^{2}$ Novosibirsk Institute of Chemical Biology and Fundamental Medicine, Siberian Branch of the Russian Academy of Sciences, 630090, Russia, Novosibirsk, pr. Lavrentieva, 8.

${ }^{3}$ Institute of Cytology and Genetics, 10, acad. Lavrentjev Ave., 630090 Novosibirsk, Russia.

DOI: 10.19163/MedChemRussia2021-2021-293

E-mail: chomenko@nioch.nsc.ru

Tyrosyl-DNA phosphodiesterase 1 (Tdp1) is a promising inhibition target for the development of new chemosensitizing agents due to its ability to remove DNA damage caused by topoisomerase 1 (Top1) poisons, such as topotecan. We have synthesized new arylcoumarin (neoflavonoid) derivatives with monoterpenoid substituents for testing their inhibitory activity against Tdp1, and studying the synergistic effect with topotecan, a clinically-important Top1 inhibitor, in in vivo experiments. Substituted coumarins were prepared by the reaction of monoterpenoid bromides with coumarins synthesized by the Pechmann condensation of resorcinol with $\beta$-ketoesters.

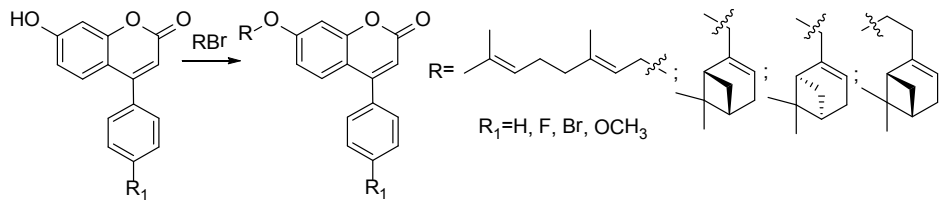

Our results showed that arylcoumarin-monoterpenoid hybrids are potent Tdp1 inhibitors with $\mathrm{IC}_{50}$ values in the submicromolar range [1]. In vivo experiments with mice were revealed that the derivative of geraniol and 4-arylcoumarin $\left(R_{1}=F\right)\left(I C_{50}\right.$ $0.62 \mu \mathrm{M}$ ) induced a significant increase in the antitumor effect of topotecan on the Krebs-2 ascites tumor model. In addition, these compounds presented the good physicchemical properties required for oral bioavailability. Thus, this type of arylcoumarinmonoterpenoid hybrids represents an excellent starting point for the further development of adjuvant therapy against cancer in combination with Top 1 poisons.

This work was supported by the Russian Science Foundation (grant 19-13-00040).

\section{References}

[1] T. M. Khomenko, A. Zakharenko, A. Chepanova at al. Int. J. Mol. Sci. 2020, 21, 126. 